\title{
Different consequences of the treatment of osteoarthritis in gastrointestinal comorbidity with exocrine pancreatic insufficiency
}

\author{
IRYNA M. HALABITSKA ${ }^{A-G}$, LILIYA S. BABINETSA-G
}

ORCID ID: 0000-0002-9028-7230

Department of Primary Health Care and General Practice of Family Medicine, I. Horbachevsky Ternopil National Medical University, Ternopil, Ukraine

A - Study Design, B - Data Collection, C - Statistical Analysis, D - Data Interpretation, E - Manuscript Preparation, F - Literature Search, G - Funds Collection

Summary Background. Patients with OA require attention of specialists due to the high integrative risk of acute conditions caused by the use of drugs that successfully treat chronic pain, but lead to gastrointestinal complications .

Objectives. The aim of the study was to evaluate the effect of anti-inflammatory treatment of primary osteoarthritis on joint and digestive functions, as well as the trophological status of patients with osteoarthritis under conditions of comorbidity with a pathology of the gastrointestinal tract.

Material and methods. The study included 87 patients with primary osteoarthritis in comorbidity with exocrine pancreatic insufficiency in gastrointestinal diseases without exacerbation. It investigated the symptoms of osteoarthritis, fecal $\alpha$-elastase levels, the CO program, the GSRS questionnaire, and the parameters of the trophological status. The studied parameters were measured before and after six weeks after the start of treatment.

Results. The use of NSAIDs contributes to regression of primary osteoarthritis symptoms according to the indices of the VAS, WOMAC, Leken, and Harris Hip tests $(p<0.05)$, but it has a negative effect on the levels of fecal $\alpha$-elastase and the CO program score $(p<0.05)$. It also leads to a deterioration of gastrointestinal symptoms according to the GSRS questionnaire $(p<0.05)$. The trophological status of patients with primary osteoarthritis with concomitant exocrine pancreatic insufficiency worsens during anti-inflammatory joint therapy $(p<0.05)$.

Conclusions. Our research indicated a multidirectional effect of NSAIDs in patients with a comorbidity of primary osteoarthritis with exocrine insufficiency of the pancreas.

Key words: exocrine pancreatic insufficiency, non-steroidal anti-inflammatory agents, osteoarthritis.

Halabitska IM, Babinets LS. Different consequences of the treatment of osteoarthritis in gastrointestinal comorbidity with exocrine pancreatic insufficiency. Fam Med Prim Care Rev 2021; 23(4): 422-428, doi: https://doi.org/10.5114/fmpcr.2021.108207.

\section{Background}

Osteoarthritis $(\mathrm{OA})$ is a heterogeneous group of diseases of various etiologies with similar biological, morphological, and clinical signs and course, which are associated with lesions of the cartilage, the subchondral bone, the synovial membrane, the ligaments, the capsules, and the periarticular muscles [1]. Patients with $\mathrm{OA}$ require interdisciplinary attention of specialists - general practitioners, rheumatologists, gastroenterologists, orthopedists, traumatologists, and surgeons - due to the high integrative risk of acute conditions caused by the use of drugs that successfully treat chronic pain, but also lead to gastrointestinal (GIT) complications [2].

Prescribing effective therapy in patients with OA has always been a difficult problem. And since patients with OA are usually older than 40 years, and therefore have more than one comorbidity, it is important to take into account the impact of drugs on purine, carbohydrate, and lipid metabolism and the safety of usage in the treatment $[3,4]$. Lesions of the gastrointestinal tract in conjunction with a decrease in the secretory function of the pancreas are frequent pathological processes that accompany primary OA as a concomitant disease [4-7]. Pathological changes in the digestive system can develop with the treatment of primary OA, especially with long-term use of NSAIDs, administered to reduce the intensity of pain and inflammation [5-7]. The comorbidity of primary $\mathrm{OA}$ and gastrointestinal diseases complicates the choice of treatment strategies due to possible mutual interferences and the risk of side effects of drugs used in the therapy [4-7].

\section{Objectives}

The aim of the study was to evaluate the effect of anti-inflammatory treatment of primary OA on the parameters of joint and digestive functions, as well as on the trophological status under conditions of comorbidity of OA with a pathology of the GIT.

\section{Material and methods}

\section{Study population}

The study included 87 patients with primary OA (before a course of anti-inflammatory therapy for 14 days) in comorbidity with exocrine pancreatic insufficiency (EPI) in gastrointestinal diseases without exacerbation: chronic pancreatitis, chronic non-stone cholecystitis, and chronic gastroduodenitis, who were undergoing outpatient treatment at the Ternopil City Center for Primary Health Care, Ukraine, between 2019 and 2020. The mean age of the patients was $59.51 \pm 6.92$ years (from 28 to 79 years); there were 44 women $(50.57 \%)$ and 43 men (49.43\%). The control group consisted of 30 healthy people. 


\section{Study groups}

The study population was classified into three group:

- Group $1(n=30)$ included patients who had a comorbidity of $\mathrm{OA}$ and chronic pancreatitis (CP).

- Group $2(n=28)$ included patients with a comorbidity of $\mathrm{OA}$ and chronic non-stone cholecystitis, functional diseases of the gallbladder, and the biliary system.

- $\quad$ Group $3(n=29)$ included patients with OA and chronic gastroduodenitis.

\section{Inclusion criteria}

Patients meeting the following criteria were eligible for inclusion in this study:

1) age over 18 years,

2) gender: patients of both sexes.

\section{Exclusion criteria}

The exclusion criteria were cancer, acute and exacerbating chronic pathologies of vital organs, severe diabetes mellitus (DM), type 1 diabetes, active gastric and duodenal ulcers, viral hepatitis and liver cirrhosis, Crohn's disease, nonspecific ulcerative colitis, and cystic fibrosis.

\section{Case confirmation}

The diagnosis of OA was carried out on the basis of diagnostic criteria of the Osteoarthritis Research Society International (OARSI), 2019, the American Association of Rheumatologists (ACR), 2020, and the European League Against Rheumatism (EULAR), 2017.

\section{Selection of the control group}

The healthy individuals in the control group were selected from Ternopil City Center for Primary Health Care in Ukraine. All the participants in the control group were free from $\mathrm{OA}$ and GIT pathology.

\section{Clinical examination}

Examination of the joints included palpation and objective assessment of pain at rest and during movement according to VAS. OA symptoms were also assessed by the Leken index, the WOMAC (Western Ontario and McMaster University) questionnaire, and the Harris Hip test [7-10]. X-ray examination was performed using KRD-50 Indiak-02 and RUM-20-2P2 X-ray equipment. The radiological stages of OA were evaluated according to the classification of J.H. Kellgren and J.S. Lawrence.

\section{Laboratory investigations}

Fecal $\alpha$-elastase content was measured to assess EPI. Fecal $\alpha$-elastase was measured by enzyme-linked immunosorbent assay using standard BIOSERV ELASTASE 1-ELISA kits. Also, to determine the presence and severity of reduction of exocrine pancreatic function and concomitant enterocolitis, the $\mathrm{CO}$ program was evaluated on a five-point scale, where the following pathological features were taken into account (scored as one point each): a high value; the presence of undigested fats (steatorrhea) in the form of neutral fats; the presence of digested fiber and starch in the stool (amilorea); a significant amount of mucus and leukocytes as evidence of an inflammatory process in the intestine; and the presence of fungi, protozoa, and helminths and their products.

To assess gastroenterological symptoms, we used the GSRS (Gastrointestinal Symptom Rating Scale) questionnaire, which contains five scores: abdominal pain (AP), indigestive syndrome (IS), diarrheal syndrome (DS), constipation syndrome (CS), and gastroesophageal reflux syndrome (RS).
Other biochemical parameters were also measured: bilirubin, ALT, and AST. Bilirubin was estimated by the colorimetric method. ALT and AST levels were measured by the Reitman-Frankel method.

Some parameters of the trophological status of patients in the blood serum were also assessed. Magnesium, calcium, and iron levels were measured by the colorimetric method. Levels of zinc, selenium, vitamins $A, E$, and $K$ were measured using the spectrophotometric method.

\section{Treatment of patients}

The patients in all the study groups received a 14-day course of NSAIDs which are officially recommended for the treatment of pain in primary OA (Meloxicam $15 \mathrm{mg} /$ day, Nimesulide 200 $\mathrm{mg} /$ day, Diclofenac sodium $150 \mathrm{mg} /$ day). The patients were not classified according to the selected NSAID. The studied parameters were measured before and after six weeks from the start of treatment.

\section{Ethical considerations}

The materials of the clinical study were considered at a meeting of the commission on bioethics of the I. Horbachevsky Ternopil National Medical University Minutes № 60 on 01 September 2020. The research was carried out in accordance with the Code of Ethics of the World Medical Association (Declaration of Helsinki). All the patients signed informed consent to participate in the study.

\section{Statistical analysis}

The conformity of the data distribution to the law of normal distribution was checked by the Kolmogorov-Smirnov test. The arithmetic mean and standard error $(\mathrm{M} \pm \mathrm{m})$ were used to describe the data in the normal distribution. We used nonparametric statistical methods to compare the groups, namely the Mann-Whitney $U$ test (for independent groups) and the Wilcoxon test (for dependent groups). We used Microsoft Exel 2016 (Microsoft) software for a personal computer and STATISTICA ${ }^{\circledR} 8.0$ (StatSoft Inc. USA) software for statistical analysis and data processing.

\section{Results}

Analysis of the obtained indicators of objective assessment of pain at rest and during movement according to the VAS, Leken index, the WOMAC questionnaire, and Harris Hip tests carried out before the treatment did not show a statistically significant difference between the indicators. However, in all three groups after the treatment, there was a statistically significant positive trend in all the indicators of OA symptoms $(p<0.05)$, which points to the feasibility of prescribing NSAIDs for the treatment of patients with primary OA (Table 1 ).

Fecal $\alpha$-elastase levels were also analyzed before and after treatment in patients with primary $\mathrm{OA}$ in comparison with the groups with exocrine pancreatic insufficiency (EPI). The lowest level of fecal $\alpha$-elastase was found in group 1 (with CP); whereas the statistically significant highest level of fecal $\alpha$-elastase was observed in group 3 (with gastroduodenitis) $(p<0.05)$. Moderate EPI was detected in all groups. The highest statistically significant score of the $\mathrm{CO}$ program was also found in the $1^{\text {st }}$ group of patients, and the lowest score was noted in the $3^{\text {rd }}$ group $(p<0.05)$. After treatment, a statistically significant decrease in the level of fecal $\alpha$-elastase was observed in all groups, and in the $1^{\text {st }}$ group the values dropped to the level of severe EPI. This proved the negative effect of NSAIDs on the severity of EPI $(p<0.05)$. There was also a statistically significant increase in the CO program in all groups $(p<0.05)$, which indicates a negative effect of NSAIDs on digestive function in patients with OA in comorbidity with EPI (Table 2). 


\begin{tabular}{|c|c|c|c|c|c|c|c|}
\hline \multirow[t]{3}{*}{ OA Symptom } & \multicolumn{7}{|c|}{ Comparison group } \\
\hline & \multirow{2}{*}{$\begin{array}{l}\text { Control } \\
(n=30)\end{array}$} & \multicolumn{2}{|c|}{$1^{\text {st }}$ group $(n=30)$} & \multicolumn{2}{|c|}{$2^{\text {nd }}$ group ( $\left.n=28\right)$} & \multicolumn{2}{|c|}{$3^{\text {rd }}$ group $(n=29)$} \\
\hline & & \begin{tabular}{|l} 
Before \\
treatment
\end{tabular} & \begin{tabular}{|l|} 
After \\
treatment
\end{tabular} & \begin{tabular}{|l|} 
Before \\
treatment
\end{tabular} & \begin{tabular}{|l|} 
After \\
treatment \\
\end{tabular} & \begin{tabular}{|l|} 
Before \\
treatment
\end{tabular} & \begin{tabular}{|l} 
After \\
treatment
\end{tabular} \\
\hline $\begin{array}{l}\text { VAS index, } \\
\text { at rest, }(\mathrm{mm})\end{array}$ & $1.11 \pm 0.12$ & $\begin{array}{l}26.84 \pm 3.56 \\
p_{1-2}<0.05\end{array}$ & $\begin{array}{l}19.36 \pm 2.54 \\
p_{2-3}<0.05\end{array}$ & $\begin{array}{l}27.01 \pm 2.97 \\
p_{1-4}<0.05\end{array}$ & $\begin{array}{l}19.41 \pm 2.58 \\
p_{4-5}<0.05\end{array}$ & $\begin{array}{l}26.99 \pm 2.49 \\
p_{1-6}<0.05\end{array}$ & $\begin{array}{l}19.31 \pm 2.79 \\
p_{6-7}<0.05\end{array}$ \\
\hline $\begin{array}{l}\text { VAS index, move- } \\
\text { ment, }(\mathrm{mm})\end{array}$ & $2.12 \pm 0.43$ & $\begin{array}{l}38.81 \pm 4.41 \\
p_{1-2}<0.05\end{array}$ & $\begin{array}{l}31.78 \pm 1.67 \\
p_{2-3}<0.05\end{array}$ & $\begin{array}{l}38.97 \pm 3.89 \\
p_{1-4}<0.05\end{array}$ & $\begin{array}{l}31.59 \pm 1.95 \\
p_{4-5}<0.05\end{array}$ & $\begin{array}{l}39.11 \pm 3.66 \\
p_{1-6}<0.05\end{array}$ & $\begin{array}{l}30.99 \pm 2.01 \\
p_{6-7}<0.05\end{array}$ \\
\hline $\begin{array}{l}\text { WOMAC index, pain, } \\
\text { score }\end{array}$ & $0.79 \pm 0.09$ & $\begin{array}{l}16.65 \pm 1.87 \\
p_{1-2}<0.05\end{array}$ & $\begin{array}{l}11.26 \pm 1.47 \\
p_{2-3}<0.05\end{array}$ & $\begin{array}{l}17.01 \pm 1.78 \\
p_{1-4}<0.05\end{array}$ & $\begin{array}{l}11.59 \pm 1.21 \\
p_{4-5}<0.05\end{array}$ & $\begin{array}{l}16.94 \pm 2.09 \\
p_{1-6}<0.05\end{array}$ & $\begin{array}{l}11.68 \pm 1.89 \\
p_{6-7}<0.05\end{array}$ \\
\hline $\begin{array}{l}\text { WOMAC index, stiff- } \\
\text { ness, score }\end{array}$ & $0.12 \pm 0.02$ & $\begin{array}{l}5.52 \pm 0.89 \\
p_{1-2}<0.05\end{array}$ & $\begin{array}{l}4.18 \pm 0.03 \\
p_{2-3}<0.05\end{array}$ & $\begin{array}{l}5.67 \pm 0.83 \\
p_{1-4}<0.05\end{array}$ & $\begin{array}{l}4.19 \pm 0.12 \\
p_{4-5}<0.05\end{array}$ & $\begin{array}{l}5.69 \pm 0.97 \\
p_{1-6}<0.05\end{array}$ & $\begin{array}{l}4.25 \pm 0.18 \\
p_{6-7}<0.05\end{array}$ \\
\hline $\begin{array}{l}\text { WOMAC index, func- } \\
\text { tional insufficiency, } \\
\text { score }\end{array}$ & $1.15 \pm 0.03$ & $\begin{array}{l}43.63 \pm 3.19 \\
p_{1-2}<0.05\end{array}$ & $\begin{array}{l}37.95 \pm 2.03 \\
p_{2-3}<0.05\end{array}$ & $\begin{array}{l}43.69 \pm 3.68 \\
p_{1-4}<0.05\end{array}$ & $\begin{array}{l}38.12 \pm 2.76 \\
p_{4-5}<0.05\end{array}$ & $\begin{array}{l}44.02 \pm 3.11 \\
p_{1-6}<0.05\end{array}$ & $\begin{array}{l}37.69 \pm 2.49 \\
p_{6-7}<0.05\end{array}$ \\
\hline $\begin{array}{l}\text { WOMAC index, total, } \\
\text { score }\end{array}$ & $2.38 \pm 0.05$ & $\begin{array}{l}72.73 \pm 5.12 \\
p_{1-2}<0.05\end{array}$ & $\begin{array}{l}63.32 \pm 3.31 \\
p_{2-3}<0.05\end{array}$ & $\begin{array}{l}73.67 \pm 4.99 \\
p_{1-4}<0.05\end{array}$ & $\begin{array}{l}63.75 \pm 3.99 \\
p_{4-5}<0.05\end{array}$ & $\begin{array}{l}71.59 \pm 3.98 \\
p_{1-6}<0.05\end{array}$ & $\begin{array}{l}64.02 \pm 4.01 \\
p_{6-7}<0.05\end{array}$ \\
\hline Leken index, score & $0.21 \pm 0.04$ & $\begin{array}{l}6.81 \pm 0.98 \\
p_{1-2}<0.05\end{array}$ & $\begin{array}{l}5.12 \pm 0.97 \\
p_{2-3}<0.05\end{array}$ & $\begin{array}{l}6.79 \pm 0.97 \\
p_{1-4}<0.05\end{array}$ & $\begin{array}{l}5.13 \pm 1.02 \\
p_{4-5}<0.05\end{array}$ & $\begin{array}{l}6.88 \pm 1.02 \\
p_{1-6}<0.05\end{array}$ & $\begin{array}{l}5.16 \pm 1.01 \\
p_{6-7}<0.05\end{array}$ \\
\hline $\begin{array}{l}\text { Harris Hip test index, } \\
\text { score }\end{array}$ & $1.31 \pm 0.11$ & $\begin{array}{l}64.41 \pm 3.79 \\
p_{1-2}<0.05\end{array}$ & $\begin{array}{l}57.32 \pm 2.65 \\
p_{2-3}<0.05\end{array}$ & $\begin{array}{l}64.67 \pm 3.88 \\
p_{1-4}<0.05\end{array}$ & $\begin{array}{l}57.98 \pm 2.68 \\
p_{4-5}<0.05\end{array}$ & $\begin{array}{l}65.11 \pm 3.98 \\
p_{1-6}<0.05\end{array}$ & $\begin{array}{l}58.11 \pm 3.21 \\
p_{6-7}<0.05\end{array}$ \\
\hline
\end{tabular}

$p_{1-2^{\prime}}, p_{1-4^{\prime}} p_{1-6}$-statistically significant difference between the groups in relation to the control group; $p_{2-3^{\prime}}, p_{4-5}, p_{6-7}-$ statistically significant difference in relation to their group before treatment.

\begin{tabular}{|c|c|c|c|c|c|c|c|}
\hline \multirow[t]{3}{*}{ EPI indicator } & \multicolumn{7}{|c|}{ Comparison group } \\
\hline & \multirow{2}{*}{$\begin{array}{l}\text { Control } \\
(n=30)\end{array}$} & \multicolumn{2}{|c|}{$1^{\text {st }}$ group $(n=30)$} & \multicolumn{2}{|c|}{$2^{\text {nd }}$ group $(n=28)$} & \multicolumn{2}{|c|}{$3^{\text {rd }}$ group $(n=29)$} \\
\hline & & $\begin{array}{l}\text { Before } \\
\text { treatment }\end{array}$ & $\begin{array}{l}\text { After } \\
\text { treatment }\end{array}$ & $\begin{array}{l}\text { Before } \\
\text { treatment }\end{array}$ & \begin{tabular}{|l|}
$\begin{array}{l}\text { After } \\
\text { treatment }\end{array}$ \\
\end{tabular} & $\begin{array}{l}\text { Before } \\
\text { treatment }\end{array}$ & \begin{tabular}{|l} 
After \\
treatment
\end{tabular} \\
\hline $\begin{array}{l}\text { Fecal } \alpha \text {-elastase, } \\
\mathrm{mcg} / \mathrm{g}\end{array}$ & $215.7 \pm 5.32$ & $\begin{array}{l}69.72 \pm 3.71 \\
p_{1-2}<0.05\end{array}$ & $\begin{array}{l}58.36 \pm 2.54 \\
p_{2-3}<0.05\end{array}$ & $\begin{array}{l}81.54 \pm 2.69 \\
p_{1-4}<0.05 \\
p_{2-4}<0.05\end{array}$ & $\begin{array}{l}73.41 \pm 1.68 \\
p_{4-5}<0.05\end{array}$ & $\begin{array}{l}93.35 \pm 3.78 \\
p_{1-6}<0.05 \\
p_{4-6}<0.05\end{array}$ & $\begin{array}{l}85.21 \pm 2.87 \\
p_{6-7}<0.05\end{array}$ \\
\hline CO program, score & $0.86 \pm 0.03$ & $\begin{array}{l}3.81 \pm 0.09 \\
p_{1-2}<0.05\end{array}$ & $\begin{array}{l}4.18 \pm 0.07 \\
p_{2-3}<0.05\end{array}$ & $\begin{array}{l}3.11 \pm 0.08 \\
p_{1-4}<0.05 \\
p_{2-4}<0.05\end{array}$ & $\begin{array}{l}3.98 \pm 0.05 \\
p_{4-5}<0.05\end{array}$ & $\begin{array}{l}2.67 \pm 0.06 \\
p_{1-6}<0.05 \\
p_{4-6}<0.05\end{array}$ & $\begin{array}{l}3.15 \pm 0.04 \\
p_{6-7}<0.05\end{array}$ \\
\hline
\end{tabular}

$p_{1-2^{\prime}}, p_{1-4^{4}}, p_{1-6}$-statistically significant difference between the groups in relation to the control group; $p_{2-3^{3}}, p_{4-55^{\prime}} p_{6-7}-$ statistically significant difference in relation to their group before treatment; $p_{2-4}, p_{4-6}$-statistically significant difference for the $2^{\text {nd }}$ and $3^{\text {rd }}$ groups, respectively.

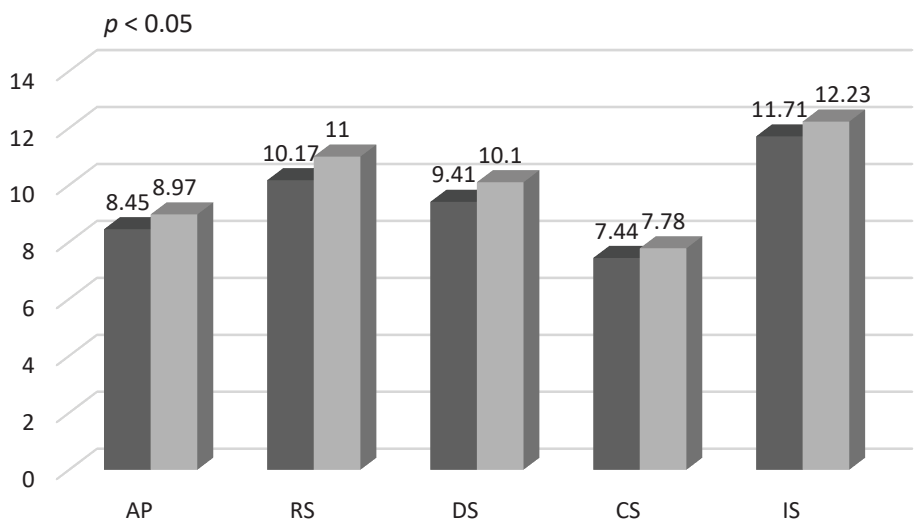

Before treatment $\quad$ After treatment
Figure 1. Changes in gastroenterological symptoms on the scales of the GSRS questionnaire in the $1^{\text {st }}$ group of patients before and after treatment
The indicators of the GSRS questionnaire were analyzed to assess the gastroenterological symptoms before treatment (Figures $1-3)$.

The most pronounced statistically significant gastroenterological symptoms on the scales of the GSRS questionnaire were found in the $1^{\text {st }}$ group of patients with comorbidity of OA and CP $(p<0.05)$. There was no statistically significant difference between the $2^{\text {nd }}$ and $3^{\text {rd }}$ groups on the scales of the GSRS questionnaire.
After treatment, a statistically significant deterioration of gastroenterological symptoms was found on all the scales of the GSRS questionnaire in all the groups of OA patients with concomitant diseases accompanied by EPI $(p<0.05)$. This proved the negative effect of NSAIDs use on the symptoms of digestive function in OA in comorbidity with diseases with EPI.

We analyzed the effect of NSAIDs on the biochemical hepatic parameters of patients with $\mathrm{OA}$ in comorbidity with EPI. 
$p<0.05$

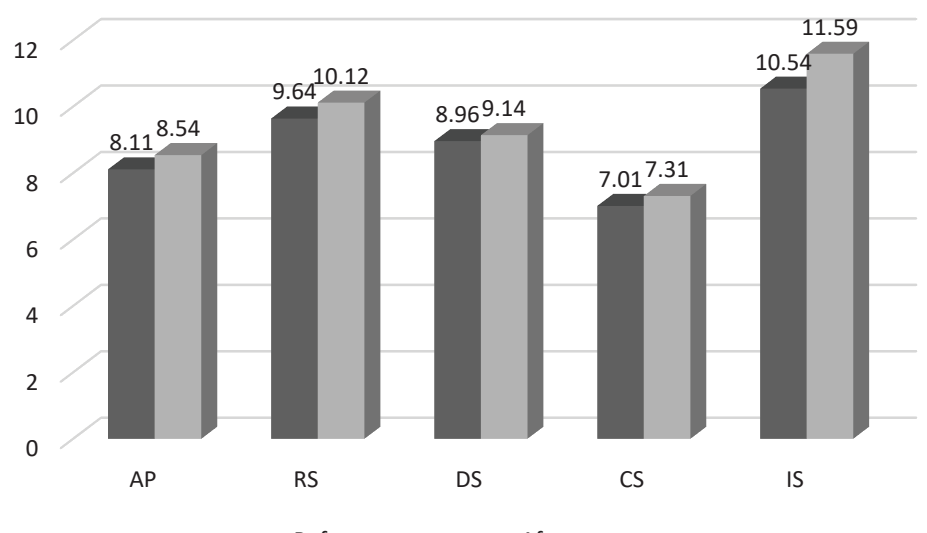

Before treatment $\quad$ After treatment

$p<0.05$

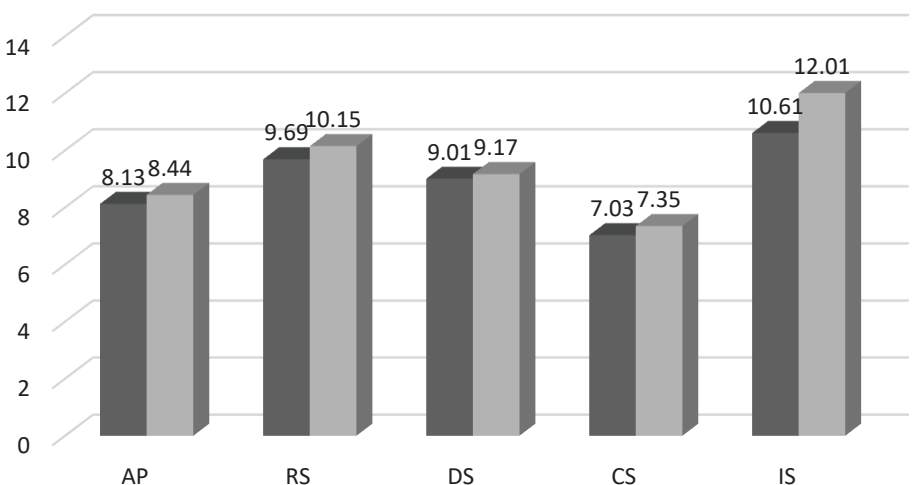

Before treatment After treatment
Figure 2. Changes in gastroenterological symptoms on the scales of the GSRS questionnaire in the $2^{\text {nd }}$ group of patients before and after treatment

Figure 3. Changes in gastroenterological symptoms on the scales of the GSRS questionnaire under the influence of anti-inflammatory treatment in the $3^{\text {rd }}$ group of patients before and after treatment

\begin{tabular}{|c|c|c|c|c|c|c|c|}
\hline \multirow{3}{*}{$\begin{array}{l}\text { Biochemical } \\
\text { hepatic index }\end{array}$} & \multicolumn{7}{|c|}{ Comparison group } \\
\hline & \multirow[t]{2}{*}{$\begin{array}{l}\text { Control } \\
(n=30)\end{array}$} & \multicolumn{2}{|l|}{\begin{tabular}{|l|}
$1^{\text {st }}$ group \\
$(n=30)$
\end{tabular}} & \multicolumn{2}{|l|}{$\begin{array}{l}2^{\text {nd }} \text { group } \\
(n=28)\end{array}$} & \multicolumn{2}{|l|}{$\begin{array}{l}3^{\text {rd }} \text { group } \\
(n=29)\end{array}$} \\
\hline & & \begin{tabular}{|l|} 
Before \\
treatment \\
\end{tabular} & \begin{tabular}{|l} 
After \\
treatment \\
\end{tabular} & $\begin{array}{l}\text { Before } \\
\text { treatment }\end{array}$ & \begin{tabular}{|l|} 
After \\
treatment \\
\end{tabular} & \begin{tabular}{|l|} 
Before \\
treatment \\
\end{tabular} & \begin{tabular}{|l} 
After \\
treatment \\
\end{tabular} \\
\hline Bilirubin, $\mu \mathrm{mol} / \mathrm{l}$ & $11.14 \pm 0.12$ & $\begin{array}{l}15.84 \pm 1.56 \\
p_{1-2}<0.05 \\
p_{2-4}<0.05\end{array}$ & $\begin{array}{l}19.67 \pm 1.52 \\
p_{2-3}<0.05\end{array}$ & $\begin{array}{l}17.31 \pm 1.31 \\
p_{1-4}<0.05\end{array}$ & $\begin{array}{l}21.65 \pm 1.28 \\
p_{4-5}<0.05\end{array}$ & $\begin{array}{l}15.42 \pm 1.32 \\
p_{1-6}<0.05 \\
p_{4-6}<0.05 \\
\end{array}$ & $\begin{array}{l}19.65 \pm 1.43 \\
p_{6-7}<0.05\end{array}$ \\
\hline ALAT, mkkat/l & $0.13 \pm 0.01$ & $\begin{array}{l}0.61 \pm 0.02 \\
p_{1-2}<0.05 \\
p_{2-4}<0.05\end{array}$ & $\begin{array}{l}0.77 \pm 0.03 \\
p_{2-3}<0.05\end{array}$ & $\begin{array}{l}0.68 \pm 0.03 \\
p_{1-4}<0.05\end{array}$ & $\begin{array}{l}0.89 \pm 0.01 \\
p_{4-5}<0.05\end{array}$ & $\begin{array}{l}0.62 \pm 0.02 \\
p_{1-6}<0.05 \\
p_{4-6}<0.05 \\
\end{array}$ & $\begin{array}{l}0.74 \pm 0.01 \\
p_{6-7}<0.05\end{array}$ \\
\hline AsAT, mkkat/l & $0.18 \pm 0.01$ & $\begin{array}{l}0.69 \pm 0.03 \\
p_{1-2}<0.05 \\
p_{2-4}<0.05\end{array}$ & $\begin{array}{l}0.76 \pm 0.04 \\
p_{2-3}<0.05\end{array}$ & $\begin{array}{l}0.75 \pm 0.03 \\
p_{1-4}<0.05\end{array}$ & $\begin{array}{l}0.79 \pm 0.01 \\
p_{4-5}<0.05\end{array}$ & $\begin{array}{l}0.67 \pm 0.02 \\
p_{1-6}<0.05 \\
p_{4-6}<0.05\end{array}$ & $\begin{array}{l}0.75 \pm 0.02 \\
p_{6-7}<0.05\end{array}$ \\
\hline
\end{tabular}

$p_{1-2}, p_{1-1}, p_{1-5}$ - statistically significant difference between the groups in relation to the control group; $p_{2-3}, p_{4-5}, p_{6-7}-$ statistically significant difference in relation to their group before treatment; $p_{2-4^{\prime}} p_{4-6}$-statistically significant difference for the $1^{\text {st }}$ and $3^{\text {rd }}$ groups, respectively.

\begin{tabular}{|c|c|c|c|c|c|c|c|}
\hline \multirow{3}{*}{$\begin{array}{l}\text { Indicator serum } \\
\text { minerals and } \\
\text { vitamins }\end{array}$} & \multicolumn{7}{|c|}{ Comparison group } \\
\hline & \multirow{2}{*}{$\begin{array}{l}\text { Control } \\
(n=30)\end{array}$} & \multicolumn{2}{|c|}{$1^{\text {st }}$ group $(n=30)$} & \multicolumn{2}{|c|}{$2^{\text {nd }}$ group $(n=28)$} & \multicolumn{2}{|c|}{$3^{\text {rd }}$ group $(n=29)$} \\
\hline & & $\begin{array}{l}\text { Before } \\
\text { treatment }\end{array}$ & $\begin{array}{l}\text { After } \\
\text { treatment }\end{array}$ & $\begin{array}{l}\text { Before } \\
\text { treatment }\end{array}$ & $\begin{array}{l}\text { After } \\
\text { treatment }\end{array}$ & $\begin{array}{l}\text { Before } \\
\text { treatment }\end{array}$ & $\begin{array}{l}\text { After } \\
\text { treatment }\end{array}$ \\
\hline $\begin{array}{l}\text { Magnesium, } \\
\mathrm{mmol} / \mathrm{l}\end{array}$ & $0.98 \pm 0.02$ & $\begin{array}{l}0.74 \pm 0.03 \\
p_{1-2}<0.05\end{array}$ & $\begin{array}{l}0.67 \pm 0.02 \\
p_{2-3}<0.05\end{array}$ & $\begin{array}{l}0.82 \pm 0.05 \\
p_{1-4}<0.05 \\
p_{2-4}<0.05\end{array}$ & $\begin{array}{l}0.74 \pm 0.04 \\
p_{4-5}<0.05\end{array}$ & $\begin{array}{l}0.83 \pm 0.02 \\
p_{1-6}<0.05 \\
p_{4-6}<0.05\end{array}$ & $\begin{array}{l}0.75 \pm 0.03 \\
p_{6-7}<0.05\end{array}$ \\
\hline Calcium, mmol/l & $2.39 \pm 0.11$ & $\begin{array}{l}2.21 \pm 0.02 \\
p_{1-2}<0.05\end{array}$ & $\begin{array}{l}2.17 \pm 0.03 \\
p_{2-3}<0.05\end{array}$ & $\begin{array}{l}2.29 \pm 0.03 \\
p_{1-4}<0.05 \\
p_{2-4}<0.05\end{array}$ & $\begin{array}{l}2.23 \pm 0.14 \\
p_{4-5}<0.05\end{array}$ & $\begin{array}{l}2.29 \pm 0.12 \\
p_{1-6}<0.05 \\
p_{4-6}<0.05\end{array}$ & $\begin{array}{l}2.16 \pm 0.09 \\
p_{6-7}<0.05\end{array}$ \\
\hline
\end{tabular}




\begin{tabular}{|c|c|c|c|c|c|c|c|}
\hline \multirow{3}{*}{$\begin{array}{l}\text { Indicator serum } \\
\text { minerals and } \\
\text { vitamins }\end{array}$} & \multicolumn{7}{|c|}{ Comparison group } \\
\hline & \multirow{2}{*}{$\begin{array}{l}\text { Control } \\
(n=30)\end{array}$} & \multicolumn{2}{|c|}{$1^{\text {st }}$ group $(n=30)$} & \multicolumn{2}{|c|}{$2^{\text {nd }}$ group $(n=28)$} & \multicolumn{2}{|c|}{$3^{\text {rd }}$ group $(n=29)$} \\
\hline & & \begin{tabular}{|l|} 
Before \\
treatment
\end{tabular} & \begin{tabular}{|l} 
After \\
treatment
\end{tabular} & \begin{tabular}{|l|} 
Before \\
treatment
\end{tabular} & $\begin{array}{l}\text { After } \\
\text { treatment }\end{array}$ & $\begin{array}{l}\text { Before } \\
\text { treatment }\end{array}$ & $\begin{array}{l}\text { After } \\
\text { treatment }\end{array}$ \\
\hline Iron, $\mu \mathrm{mol} / \mathrm{I}$ & $27.11 \pm 1.21$ & $\begin{array}{l}19.67 \pm 0.03 \\
p_{1-2}<0.05\end{array}$ & $\begin{array}{l}14.88 \pm 0.04 \\
p_{2-3}<0.05\end{array}$ & $\begin{array}{l}22.31 \pm 0.11 \\
p_{1-4}<0.05 \\
p_{2-4}<0.05\end{array}$ & $\begin{array}{l}16.12 \pm 0.13 \\
p_{4-5}<0.05\end{array}$ & $\begin{array}{l}22.39 \pm 0.18 \\
p_{1-6}<0.05 \\
p_{4-6}<0.05\end{array}$ & $\begin{array}{l}16.28 \pm 0.14 \\
p_{6-7}<0.05\end{array}$ \\
\hline Zinc, $\mu \mathrm{mol} / \mathrm{I}$ & $119.43 \pm 2.19$ & $\begin{array}{l}87.51 \pm 4.32 \\
p_{1-2}<0.05\end{array}$ & $\begin{array}{l}74.15 \pm 3.21 \\
p_{2-3}<0.05\end{array}$ & $\begin{array}{l}93.13 \pm 1.32 \\
p_{1-4}<0.05 \\
p_{2-4}<0.05\end{array}$ & $\begin{array}{l}86.32 \pm 2.56 \\
p_{4-5}<0.05\end{array}$ & $\begin{array}{l}93.54 \pm 1.21 \\
p_{1-6}<0.05 \\
p_{4-6}<0.05\end{array}$ & $\begin{array}{l}87.02 \pm 1.12 \\
p_{6-7}<0.05\end{array}$ \\
\hline Selenium, $\mu \mathrm{mol} / \mathrm{l}$ & $1.19 \pm 0.13$ & $\begin{array}{l}0.99 \pm 0.01 \\
p_{1-2}<0.05\end{array}$ & $\begin{array}{l}0.76 \pm 0.02 \\
p_{2-3}<0.05\end{array}$ & $\begin{array}{l}1.05 \pm 0.02 \\
p_{1-4}<0.05 \\
p_{2-4}<0.05\end{array}$ & $\begin{array}{l}0.91 \pm 0.03 \\
p_{4-5}<0.05\end{array}$ & $\begin{array}{l}1.07 \pm 0.05 \\
p_{1-6}<0.05 \\
p_{4-6}<0.05\end{array}$ & $\begin{array}{l}0.89 \pm 0.05 \\
p_{6-7}<0.05\end{array}$ \\
\hline Albumin, $\mathrm{g} / \mathrm{I}$ & $49.21 \pm 2.56$ & $\begin{array}{l}39.43 \pm 1.53 \\
p_{1-2}<0.05\end{array}$ & $\begin{array}{l}36.11 \pm 0.97 \\
p_{2-3}<0.05\end{array}$ & $\begin{array}{l}42.11 \pm 1.15 \\
p_{1-4}<0.05 \\
p_{2-4}<0.05\end{array}$ & $\begin{array}{l}39.21 \pm 1.67 \\
p_{4-5}<0.05\end{array}$ & $\begin{array}{l}42.23 \pm 1.16 \\
p_{1-6}<0.05 \\
p_{4-6}<0.05\end{array}$ & $\begin{array}{l}40.02 \pm 1.15 \\
p_{6-7}<0.05\end{array}$ \\
\hline Vitamin A, $\mu \mathrm{mol} / \mathrm{I}$ & $2.45 \pm 0.22$ & $\begin{array}{l}1.87 \pm 0.02 \\
p_{1-2}<0.05\end{array}$ & $\begin{array}{l}1.23 \pm 0.02 \\
p_{2-3}<0.05\end{array}$ & $\begin{array}{l}1.95 \pm 0.03 \\
p_{1-4}<0.05 \\
p_{2-4}<0.05\end{array}$ & $\begin{array}{l}1.43 \pm 0.07 \\
p_{4-5}<0.05\end{array}$ & $\begin{array}{l}1.96 \pm 0.02 \\
p_{1-6}<0.05 \\
p_{4-6}<0.05\end{array}$ & $\begin{array}{l}1.48 \pm 0.05 \\
p_{6-7}<0.05\end{array}$ \\
\hline Vitamin $E, \mu \mathrm{mol} / \mathrm{l}$ & $31.43 \pm 1.78$ & $\begin{array}{l}15.32 \pm 0.96 \\
p_{1-2}<0.05\end{array}$ & $\begin{array}{l}13.21 \pm 0.43 \\
p_{2-3}<0.05\end{array}$ & $\begin{array}{l}19.23 \pm 1.02 \\
p_{1-4}<0.05 \\
p_{2-4}<0.05\end{array}$ & $\begin{array}{l}16.11 \pm 1.02 \\
p_{4-5}<0.05\end{array}$ & $\begin{array}{l}19.27 \pm 1.11 \\
p_{1-6}<0.05 \\
p_{4-6}<0.05\end{array}$ & $\begin{array}{l}16.21 \pm 1.16 \\
p_{6-7}<0.05\end{array}$ \\
\hline Vitamin $\mathrm{K}, \mathrm{nmol} / \mathrm{l}$ & $4.91 \pm 0.98$ & $\begin{array}{l}3.69 \pm 0.16 \\
p_{1-2}<0.05\end{array}$ & $\begin{array}{l}2.87 \pm 0.07 \\
p_{2-3}<0.05\end{array}$ & $\begin{array}{l}4.01 \pm 0.15 \\
p_{1-4}<0.05 \\
p_{2-4}<0.05\end{array}$ & $\begin{array}{l}3.71 \pm 0.17 \\
p_{4-5}<0.05\end{array}$ & $\begin{array}{l}4.02 \pm 0.04 \\
p_{1-6}<0.05 \\
p_{4-6}<0.05\end{array}$ & $\begin{array}{l}3.69 \pm 0.19 \\
p_{6-7} 0.05\end{array}$ \\
\hline
\end{tabular}

$p_{1-2^{\prime}} p_{1-4^{\prime}} p_{1-6}-$ statistically significant difference between the groups in relation to the control group; $p_{2-3^{\prime}}, p_{4-5}, p_{6-7}-$ statistically significant difference in relation to their group before treatment; $p_{2-4}, p_{4-6}$-statistically significant difference for the $2^{\text {nd }}$ and $3^{\text {rd }}$ groups, respectively.

The highest content of bilirubin, ALT, and AST before treatment was found in the serum of patients with OA from group 2 (with hepatobiliary disorders) $(p<0.05)$. After treatment, patients in all the groups had increased bilirubin, AST, and ALT $(p<0.05)$. In the $2^{\text {nd }}$ group, these biochemical parameters exceeded the norm, which proved the negative impact of NSAIDs on liver function (Table 3).

The parameters of the trophological status of patients with OA with a comorbidity and EPI were studied. There was a statistically significant decrease in all the studied indicators of the trophological status of patients compared with the control group $(p<0.05)$. The parameters of the trophological status were the lowest in the $1^{\text {st }}$ group compared to the $2^{\text {nd }}$ and $3^{\text {rd }}$ groups $(p<$ 0.05 ). After treatment, there was a statistically significant decrease in the level of macronutrients, micronutrients, and vitamins in all the study groups. This revealed the deterioration of nutrient uptake due to the aggravation of EPI in OA patients with Gl comorbidities (Table 4).

\section{Discussion}

The development of adverse reactions after the use of NSAIDs remains an urgent problem of modern medicine. Many studies have been conducted to identify the effect of NSAIDs on the development of cardiovascular pathology in patients with OA $[11,12]$. A study was also carried out on the effect of different combinations of disease-modifying drugs and NSAIDs on the course of $O A$, reducing pain, and improving the quality of life of patients [13-15]. The influence of different NSAIDs on the level of pain syndrome in $\mathrm{OA}$ and on the condition of the cartilage was also researched and compared. The effectiveness of systemic and local NSAID use and the positive effect of NSAID use in patients with chronic pain was assessed [16-18]. Much attention has been paid to the investigation of the development of side effects with the use of NSAIDs [19-24]. The main side effects after NSAIDs which have been covered in many studies [25-32] are gastrointestinal disorders (nausea, vomiting, abdominal pain, diarrhea, constipation, flatulence, dyspepsia, dry mouth, peptic ulcers, bleeding, perforated ulcers, anorexia, elevated liver enzymes, pancreatic damage, and hepatic damage) [33-37]; neurological disorders (sleep disorders, anxiety, headache, dizziness, hot flashes, and paresthesia); disorders of the cardiovascular system (increased blood pressure, palpitations, tachycardia, hypotension, and peripheral edema) [38-40]; allergic complications (bronchospasm, shortness of breath, skin rash, the Stevens-Johnson and Lyell syndromes, photosensitization, anaphylactic reactions, facial edema, and itching) [41, 42]; disorders of the genitourinary system (nephritis or nephrotic syndrome, polyuria, menstrual disorders in women, and disorders of the prostate in men) [39]. Much attention is paid to the study of acid-dependent diseases of the gastrointestinal tract, which develop after NSAIDs use $[43,44]$. However, no studies have been found to study the effect of NSAIDs on the progression of EPI and the development of trophological disorders, especially under conditions of primary OA comorbidity with diseases accompanied by EPI.

\section{Limitations of the study}

The limitations of our study are the short study period, which does not include the long-term effects of drugs, as well as the small number of patients who were included in each group. There was also no differentiation between the effects of different NSAIDs classes included in the study on the indicators studied. However, in the future, we plan to investigate the longterm effects that may be caused by the drugs used in the study, increase the number of patients and differentiate the effect of different NSAIDs on the studied indicators, and also to study the three NSAIDs prospectively with a calculation of the number of patients needed to achieve $p$-value of less than $5 \%$. 


\section{Conclusions}

The use of NSAIDs contributes to statistically significant regression of symptoms of primary OA on the indices of the VAS, WOMAC, Leken, and Harris Hip tests $(p<0.05)$, which indicates the feasibility of NSAIDs in reducing joint pain in patients with primary OA in combination with accompanying EPI.

The use of NSAIDs for the treatment of patients with primary OA in comorbidity with diseases accompanied by EPI was followed by a statistically significant negative effect on the content of fecal $\alpha$-elastase and the CO program score $(p<0.05)$, especially in patients with concomitant $\mathrm{CP}$, which indicates adverse effects of NSAIDs on EPI in primary OA. There was also a statistically significant deterioration of gastrointestinal symptoms on the scales of the GSRS questionnaire after treatment with
NSAIDs $(p<0.05)$, which showed the negative effect of NSAIDs on comorbid gastrointestinal pathology in primary OA.

Deterioration of biochemical hepatic blood parameters in patients with primary OA was found, especially in conditions of comorbidity with diseases of the hepatobiliary system $(p<$ 0.05). Increased bilirubin, ALT, and AST indicated an adverse effect of NSAIDs on liver function, which exacerbated EPI.

Deterioration of the parameters of the trophological status of patients with primary OA occurred with concomitant EPI in the course of anti-inflammatory joint therapy $(p<0.05)$. This decrease in the content of macroelements, microelements, and vitamins under the influence of NSAIDs $(p<0.05)$ needs to be corrected, as it may have a negative impact on pathophysiological processes with subsequent negative mutual interference on the course of comorbid pathologies with EPI in primary OA.

Source of funding: This work was funded from the authors' own resources.

Conflicts of interest: The authors declare no conflicts of interest.

\section{References}

1. Osteoarthritis Guideline Recommendations for the Use of Nonpharmacologic and Pharmacologic Therapies in Osteoarthritis of the Hand, Hip, and Knee. American College of Rheumatology [serial online] 2020 [cited 6.01.2020]. Available from URL: https://www. rheumatology.org/Practice-Quality/Clinical-Support/Clinical-Practice-Guidelines.

2. Bannuru RR, Osani MC, Vaysbrot EE, et al. OARSI Guidelines for the non-surgical management of knee, hip, and polyarticular osteoarthritis. Osteoarthritis Cartilage 2019; 27(11): 1578-1589, doi: 10.1016/j.joca.2019.06.011.

3. Sakellariou G, Conaghan PG, Zhang W, et al. EULAR recommendations for the use of imaging in the clinical management of peripheral joint osteoarthritis Ann Rheum Dis 2017; 76: 1484-1494, doi: 10.1136/annrheumdis-2016-210815.

4. Babinets LS, Halabitska IM. Chronic inflammatory process and bone tissue changes in patients with osteoarthritis and exocrine pancreatic insufficiency Lekarsky Obzor 2020; 69(1): 7-10.

5. Babinets LS, Halabitska IM, Kotsaba YY, et al. The effect of the proteolisis' system activity for the trophological status of patients with osteoarthritis and exocrine insufficiency of pancreas. Wiad Lek 2018; 71(2 pt 1): 273-276.

6. Babinets LS, Kytsai KY, Kotsaba YY, et al. Improvement of the complex medical treatment for the patients with chronic biliary pancreatitis. Wiad Lek 2017; 70(2): 213-216.

7. Babinets LS, Halabitska IM. Characteristics of joint pain in patients with primary osteoarthritis and comorbid conditions with exocrine pancreatic insufficiency Lekarsky Obzor 2021; 70(2): 62-64.

8. Gandek B. Measurement properties of the Western Ontario and McMaster Universities Osteoarthritis Index: a systematic review. Arthritis Care Res (Hoboken) 2015; 67(2): 216-229, doi: 10.1002/acr.22415.

9. Buranova S, Akhmedov K, Razakova F. The importance of treatment aimed at the dynamics of Cartilage Oligomer Matrix Protein (COMP) in patients with the knee joint osteoarthritis. Am J Med Med Sci 2021; 11(2): 148-153, doi: 10.5923/j.ajmms.20211102.18

10. Nilsdotter A, Bremander A. Measures of hip function and symptoms: Harris Hip Score (HHS), Hip Disability and Osteoarthritis Outcome Score (HOOS), Oxford Hip Score (OHS), Lequesne Index of Severity for Osteoarthritis of the Hip (LISOH), and American Academy of Orthopedic Surgeons (AAOS) Hip and Knee Questionnaire Review. Arthritis Care Res (Hoboken) 2011; 63 (Suppl. 11): S200-S207, doi: 10.1002/acr.20549.

11. Kikuchi S, Togo K, Ebata N, et al. Database analysis on the relationships between nonsteroidal anti-inflammatory drug treatment variables and incidence of acute myocardial infarction in Japanese patients with osteoarthritis and chronic low back pain. Adv Ther 2021; 38(3): 1601-1613, doi: 10.1007/s12325-021-01629-6.

12. Ebata-Kogure N, Murakami A, Nozawa K, et al. Treatment and healthcare cost among patients with hip or knee osteoarthritis: a crosssectional study using a real-world claims database in Japan between 2013 and 2019. Clin Drug Investig 2020; 40(11): 1071-1084, doi: 10.1007/s40261-020-00968-6.

13. Leerling AT, Cañete AN, Ramautar AIE, et al. Sternocostoclavicular hyperostosis: positive clinical and radiological response on Pamidronate. Front Endocrinol (Lausanne) 2021; 12, doi: 10.3389/fendo.2021.621604.

14. Zhao J-L, Liang G-H, Pan J-K, et al. Network meta-analysis of oral Chinese patent medicine in treatment of knee osteoarthritis. Zhongguo Zhongyao Zazhi 2021; 46(4): 981-999, doi: 10.19540/j.cnki.cjcmm.20200721.502.

15. Pereira D, Peleteiro B, Araújo J, et al. The effect of osteoarthritis definition on prevalence and incidence estimates: a systematic review. Osteoarthritis Cartilage 2011; 19(11): 1270-1285, doi: 10.1016/j.joca.2011.08.009.

16. Leyland KM, Gates LS, Sanchez-Santos MT, et al. Knee osteoarthritis and time-to all-cause mortality in six community-based cohorts: an international meta-analysis of individual participant-level data. Aging Clin Exp Res 2021; 33(3): 529-545, doi: 10.1007/s40520-020-01762-2.

17. Maloney J, Pew S, Wie C, et al. Comprehensive review of topical analgesics for chronic pain. Curr Pain Headache Rep 2021; 25(2), doi: 10.1007/s11916-020-00923-2.

18. Dahlhamer JM, Lucas J, Zelaya C, et al. Prevalence of chronic pain and high-impact chronic pain among adults - United States, 2016. MMWR 2018; 67(36): 1001-1006, doi: 10.15585/mmwr.mm6736a2.

19. Parkoohi PI, Amirzadeh K, Mohabbati V, et al. Satisfaction with chronic pain treatment Anesth Pain Med 2015; 5(4), doi: 10.5812/ aapm.23528.

20. Selvanathan J, Pham C, Nagappa M, et al. Cognitive behavioral therapy for insomnia in patients with chronic pain - a systematic review and meta-analysis of randomized controlled trials. Sleep Med Rev 2021; 60, doi: 10.1016/j.smrv.2021.101460.

21. Mahesh G, Kumar KA, Reddanna P. Overview on the discovery and development of anti-inflammatory drugs: should the focus be on synthesis or degradation of pge2? J Inflamm Res 2021; 4: 253-263, doi: 10.2147/JIR.S278514.

22. Alimoradian A, Samimi F, Aslfalah H, et al. Piroxicam reduces acute and chronic pain response in type 1 diabetic rats. J Basic Clin Physiol Pharmacol 2021; doi: 10.1515/jbcpp-2019-0367. 
23. Guma A, Akhtar S, Najafzadeh M, et al. Ex vivo/in vitro effects of aspirin and ibuprofen, bulk and nano forms, in peripheral lymphocytes of prostate cancer patients and healthy individuals. Mutat Res 2021; 861-862, doi: 10.1016/j.mrgentox.2020.503306.

24. Dandah O, Najafzadeh M, Isreb M, et al. Aspirin and ibuprofen, in bulk and nanoforms: effects on DNA damage in peripheral lymphocytes from breast cancer patients and healthy individuals. Mutat Res Genet Toxicol Environ Mutagen 2018; 826: 41-46.

25. Malmgaard-Clausen NM, Jørgensen $\mathrm{OH}, \mathrm{H} \emptyset f$ ner $\mathrm{R}$, et al. No additive clinical or physiological effects of short-term anti-inflammatory treatment to physical rehabilitation in the early phase of human achilles tendinopathy: a randomized controlled trial. Am J Sports Med 2021, doi: 10.1177/0363546521991903.

26. Sağık BN, Osmaniye $D$, Levent $S$, et al. Design, synthesis and biological assessment of new selective COX-2 inhibitors including methyl sulfonyl moiety. Eur J Med Chem 2021; 209, doi: 10.1016/j.ejmech.2020.112918.

27. He L, Zhang S, Peng D, et al. Synthesis and evaluations of selective COX-2 inhibitory effects: Benzo[d]thiazol analogs. Bioorg Med Chem Lett 2020; 30(17), doi: 10.1016/j.bmcl.2020.127376.

28. Metwally NH, Mohamed MS. New imidazolone derivatives comprising a benzoate or sulfonamide moiety as anti-inflammatory and antibacterial inhibitors: design, synthesis, selective COX-2, DHFR and molecular-modeling study. Bioorg Chem 2020; 99, doi: 10.1016/j. bioorg.2019.103438.

29. Marino F, D'Angelo S, Masala IF, et al. Toxicological considerations in the treatment of axial spondylo-arthritis. Expert Opin Drug Metab Toxicol 2020; 16(8): 663-672, doi: 10.1080/17425255.2020.1783240.

30. Lio C, Luo J, Shen X, et al. Nardosinanone $\mathrm{N}$ suppresses LPS-induced macrophage activation by modulating the Nrf2 pathway and mPGES-1. Biochem Pharmacol 2020; 173, doi: 10.1016/j.bcp.2019.113639.

31. Choy EHS, Panayi GS. Cytokine pathways and joint inflamation in rheumatoid arthritis. N Engl J Med 2001; 344(12): 907-916, doi: 10.1056/NEJM200103223441207.

32. Sala A, Proschak E, Steinhilber D, et al. Two-pronged approach to anti-inflammatory therapy through the modulation of the arachidonic acid cascade. Biochem Pharmacol 2018; 158: 161-173, doi: 10.1016/j.bcp.2018.10.007.

33. Ton J, Perry D, Thomas B, et al. [PEER umbrella systematic review of systematic reviews management of osteoarthritis in primary care]. Can Fam Physician 2020; 66(3): E89-E98 (in French).

34. Hunter DJ, Bierma-Zeinstra S. Osteoarthritis. Lancet 2019; 393(10182): 1745-1759, doi: 10.1016/S0140-6736(19)30417-9.

35. Fening NY. Novel NSAIDs. SAJAA 2020; 26(6): S45-S48, doi: 10.36303/SAJAA.2020.26.6.S3.2536.

36. Yammine P, Moussa D, Ayrouth M, et al. Nanoencapsulation of NSAIDs Naproxen and Ibuprofen. Int J Pharm Sci and Res 2017; 8(8): 3347-3353, doi: 10.13040/IJPSR.0975-8232.8(8).3347-53.

37. Atkinson TJ, Fudin J, Jahn HL, et al. What's new in NSAID pharmacotherapy: oral agents to injectables. Pain Med 2013; 14(Suppl. 1): S11-S17, doi: 10.1111/ pme.12278.

38. Rang HP, Dale MM, Ritter JM, et al. Pharmacology. 5th ed. London: Elsevier Churchill Livingstone; 2003.

39. Atchison JW, Herndon CM, Rusie E. NSAIDs for musculoskeletal pain management: current perspectives and novel strategies to improve safety. J Manag Care Pharm 2013; 19(9 Suppl. A): S3-S19, doi: 10.18553/ jmcp.2013.19.s9.1.

40. Hassib ST, Hassan GS, El-Zaher AA, et al. Synthesis and biological evaluation of new prodrugs of etodolac and tolfenamic acid with reduced ulcerogenic potential. Eur J Pharm Sci 2019; 140: 105101, doi: 10.1016/j. ejps.2019.105101.

41. Rao P, Knaus EE. Evolution of nonsteroidal anti-inflammatory drugs (NSAIDs): cyclooxygenase (COX) inhibition and beyond. J Pharm Pharm Sci 2008; 11(2): 81-110, doi: 10.18433/j3t886.

42. SASA. South African Acute Pain Guidelines. 2nd ed. Pretoria: Medpharm Publications (Pty) Ltd; 2016.

43. Peesa JP, Yalavarthi PR, Rasheed A, et al. A perspective review on the role of novel NSAID prodrugs in the management of acute inflammation. J Acute Dis 2016; 5(5): 364-381, doi: 10.1016/j.joad.2016.08.002.

44. Blackler R, Syer S, Bolla M, et al. Gastrointestinal-sparing effects of novel NSAIDs in rats with compromised mucosal defence. PLoS ONE 2012; 7(4): e35196, doi: 10.1371/journal.pone.0035196.

Tables: 4

Figures: 4

References: 44

Received: 06.04.2021

Reviewed: 14.04 .2021

Accepted: 18.05 .2021

Address for correspondence:

Iryna M. Halabitska, PhD, MD

I. Horbachevsky Ternopil National Medical University

Ternopil

Ukraine

Tel.: +380977982893

E-mail: irynkagal@gmail.com, halabitska@tdmu.edu.ua 